\title{
A Population-Based Multivariate Analysis of the Association of County Demographic and Medical System Factors with Per Capita Pediatric Trauma Death Rates in North Carolina
}

\author{
Robert Rutledge, M.D., F.A.C.S., Chandra Y. Smith, M.S.II, \\ and Richard G. Azizkhank, M.D., F.A.C.S.
}

From the Department of Surgery, School of Medicine, University of North Carolina at Chapel Hill, Chapel Hill, North Carolina

\section{Objective}

This study analyzed the association between demographic and medical system factors and the pediatric trauma death rate in North Carolina.

\section{Summary Background Data}

Trauma is the leading cause of death in children. Various medical system factors have been suggested to reduce pediatric morbidity and mortality rates, but the association with these rates has not been tested.

\section{Methods}

Data were obtained from the North Carolina medical examiner's database. The dependent variable was the county per capita pediatric trauma death rate. Twenty-one demographic and medical system measures were selected as independent variables.

\section{Results}

Nine hundred forty-one pediatric trauma deaths from 1986 to 1989 were included in our sample. Multivariate analysis identified the variables most highly associated with the dependent variables. The presence of advanced life support (ALS) training was the only medical system factor associated significantly with pediatric trauma death rates. Trauma centers, emergency (911) telephone access, and other medical resource variables had no significant association.

\section{Conclusions}

The study confirms other reports showing that demographic factors have an important predictive association with the trauma death rate in children. Advanced life support was the only medical system resource associated significantly with pediatric trauma death rates. This study underlines the significance of pre-hospital care in the treatment of pediatric trauma. 
Trauma kills more children than any other disease and is more common among the poor and minorities, and in rural areas. ${ }^{1-4}$ Attention has been focused increasingly on pediatric trauma as a serious public health problem. As a result, various medical system changes have been suggested to decrease childhood deaths caused by injury. ${ }^{3.5}$ Child safety seats, bicycle helmet laws, seat belt laws, and the implementation of pediatric advanced life support training from emergency medical services (EMS) are some interventions to decrease morbidity and mortality of childhood injury. Unfortunately, few of these suggested interventions have been tested.

This study used a statewide, population-based database to assess the association of county demographic and medical system factors with county per capita pediatric trauma death rates. This database includes information on all trauma deaths in North Carolina. Populationbased studies to explore epidemiologic concerns can provide a more complete understanding of trauma death in children and can give insights into the possible effects of medical system factors on pediatric trauma deaths. Identifying the demographic variables that place children at greater risk for death due to injury and the medical system factors that are strongly associated with lower per capita trauma death rates may encourage progress in a number of areas. It may facilitate optimal development of trauma systems, use of resources to minimize the morbidity and mortality of injury, and development of appropriate injury prevention programs.

\section{MATERIALS AND METHODS}

\section{Data Source}

Data on the trauma deaths in the state were obtained from the North Carolina medical examiner's database. The N.C. Medical Examiner System was established in 1967 and contains all deaths due to injury (unintentional injury, suicide, and homicide). This database contains information on all 16,814 trauma-related deaths in North Carolina between 1986 to 1989, regardless of the time of death after injury. This information was corroborated by the North Carolina Office of Vital Statistics, which records all resident deaths in North Carolina. Of the 16,814 trauma-related deaths in North Carolina from 1986 to $1989,13,100$ occurred in individuals who lived in the county in which they sustained their injury. This subgroup of individuals was selected for further analysis.

Only children younger than age 15 at the time of death

Address reprint requests to Robert Rutledge, M.D., University of North Carolina at Chapel Hill, School of Medicine, Department of Surgery, CB\# 7210 Burnett-Womack, Chapel Hill, NC 27599 Accepted for publication June 7, 1993.

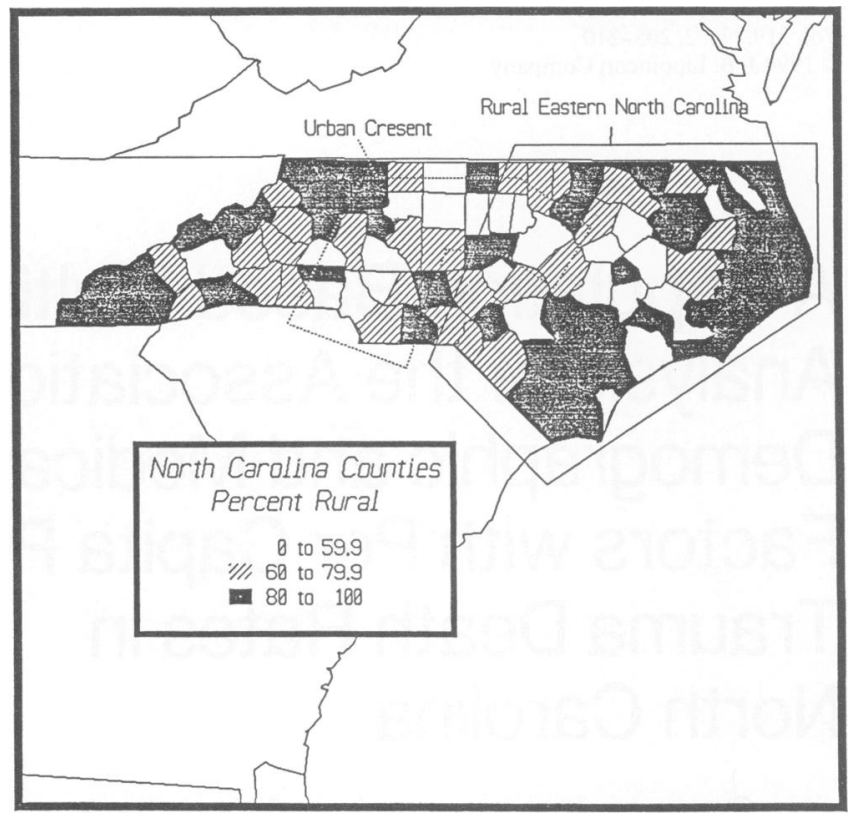

Figure 1. Rural urban distribution of North Carolina counties.

were selected for the study sample. The reasons for excluding children in the 15- to 18-year age group were as follows: children older than age 15 are more likely to be operators of motor vehicles and, unlike their younger cohorts, may be drivers in motor vehicle crashes; children older than age 15 are physically more like adults than younger children; and the causes of death in children aged 15 to 18 years are more similar to those of adults than of younger children.

\section{North Carolina Demographic Factors}

There are 100 counties in North Carolina, combining rural and urban areas (Fig. 1). For this study, a county was designated as rural if more than $60 \%$ of its population lived in open countryside or in towns with populations less than 2500 .

Studies have shown relationships between population demographics and traumatic injury. ${ }^{6-8}$ County demographic descriptors were analyzed to determine their relationships to per capita pediatric trauma death rates. Twenty-one county descriptors were selected from those available from the State Office of Management and Budget. The descriptors are listed in Table 1. To select candidate variables, a preliminary group of descriptors was chosen, based on general categories of interest. Preliminary $t$ tests were run on this group to select reasonable candidate variables for further multivariate analysis. The descriptors fell into the following categories: geographic, educational, socioeconomic, occupational, environmental, and medical factors. For this study, the North Carolina Office of Emergency Medical Services 


\section{Table 1. COUNTY DESCRIPTORS}

Geographic
Population Density
Percent rural area in county
Rural (Y/N)
Total population
Socioeconomic
Mean family income
Mean personal income
Percent non-Caucasian
Percent unemployed
Environment
Miles of primary highways
Highway exposure
Medical Resources
No. of hospital ICU beds
No. of hospital discharges
No. of ED outpatient visits
No. of general surgeons
No. of certified general surgeons
No. of blood banks
No. of neurosurgeons
No. of hospital admissions
Trauma center in county
Advanced life support in county
911 in county

(NCOEMS) definition of an advanced life support (ALS) county was used. It defines an ALS county as a county that contains one or more EMS trained at or above the emergency medical technician (EMT)-D level.

\section{Statistical Analysis}

The data were analyzed using a relational database program and an IBM-compatible computer. The file was then converted into a SAS (Statistical Analysis Systems, SAS Institute Inc., Cary, NC) data file for further analysis. The relationship of each independent variable to per capita pediatric county trauma death rates was analyzed using Pearson correlation coefficients for continuous variables and Student's $t$ test for comparing dichoto-

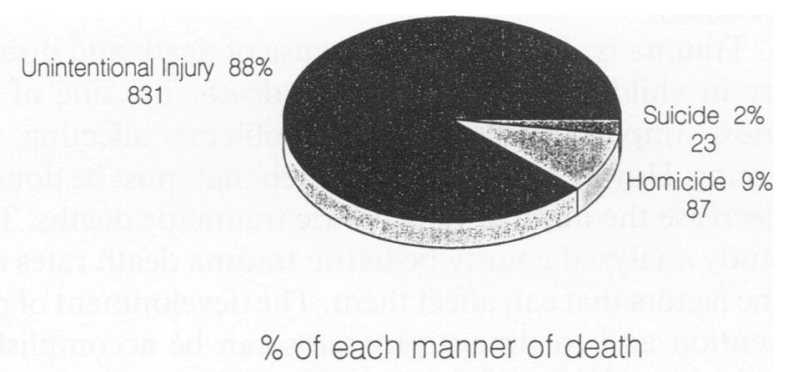

For NC 1986-1989

Figure 2. Manner of trauma deaths in children younger than 15 years.
Means of Death

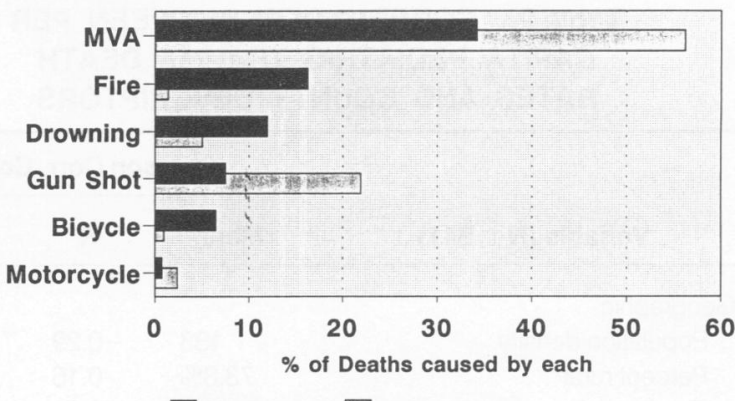

Ages 0-14 Ages 15-18

For NC 1986-1989

Figure 3. Comparison of means of death between ages 0 to 14 years and 15 to 18 years.

mous and categorical variables (i.e., 911 telephone service, presence of trauma center, and presence of ALS or basic life support). Stepwise multiple linear regression and stepwise discriminant analyses were used to derive a multivariate model to predict pediatric county trauma death rates.

\section{RESULTS}

Children younger than 15 make up $21 \%$ of North Carolina's population. There were 13,100 trauma-related deaths in North Carolina between 1986 and 1989; 7\% (941) of these deaths occurred in children younger than 15 . The mean per capita county trauma death rate was $2 / 10,000$ children per county.

Accidents $(88 \%)$ were the leading cause of death for children younger than 15 years, followed by homicide (9\%) and suicide (3\%) (Fig. 2). Motor vehicle crashes (34\%), fires $(16 \%)$, drownings $(12 \%)$, gun shot wounds (8\%), and bicycle accidents (6\%) were responsible for the majority of deaths in children younger than 15 years. The remaining $23 \%$ of deaths were a result of other events. Deaths in children age 15 to 18 years overwhelmingly were caused by motor vehicle crashes $(56 \%)$ and gun shot wounds (22\%) (Fig. 3).

Boys were over-represented, with 605 (64\%) deaths among boys and $336(36 \%)$ deaths among girls. There were 519 (55\%) deaths among Caucasians, 381 (40\%) deaths among African Americans, and 41 (4\%) deaths among individuals of other races (the majority being Native Americans). African Americans make up approximately $20 \%$ of the population of North Carolina; therefore, they are overrepresented in trauma deaths in children.

\section{Univariate Analysis of the Association of Per Capita County Pediatric Trauma Death Rates with County Demographic Variables and Medical System Factors \\ Geographic Descriptors}

Of the four geographic variables studied, two were found to be negatively correlated with per capita county 
Table 2. CORRELATION BETWEEN PER CAPITA PEDIATRIC TRAUMA DEATH RATES AND COUNTY DESCRIPTORS

\begin{tabular}{|c|c|c|c|}
\hline \multirow[b]{2}{*}{ Variable $(N=941)$} & \multicolumn{3}{|c|}{ Pearson Corr. Coeff. } \\
\hline & Mean & $\mathbf{R}$ & p Value \\
\hline \multicolumn{4}{|l|}{ Geographic } \\
\hline Population density & 133 & -0.29 & 0.014 \\
\hline Percent rural & $73.8 \%$ & 0.16 & 0.020 \\
\hline $\operatorname{Rural}(\mathrm{Y} / \mathrm{N})$ & $81 / 19^{\star}$ & 0.25 & 0.197 \\
\hline Total population & 641 & -0.21 & 0.080 \\
\hline \multicolumn{4}{|l|}{ Socioeconomic } \\
\hline Mean family income & $\$ 15,328$ & -0.20 & 0.093 \\
\hline Mean personal income & $\$ 8,850$ & -0.22 & 0.063 \\
\hline Percent non-Caucasian & $23.3 \%$ & 0.24 & 0.040 \\
\hline Percent unemployed & $2.4 \%$ & 0.22 & 0.066 \\
\hline \multicolumn{4}{|l|}{ Environment } \\
\hline Miles of primary highways & 139 & 0.16 & 0.175 \\
\hline Highway exposure & 4877 & -0.03 & 0.796 \\
\hline \multicolumn{4}{|l|}{ Medical resources } \\
\hline No. of hospital ICU beds & 12.08 & -0.23 & 0.054 \\
\hline No. of hospital discharges & 8158 & -0.22 & 0.061 \\
\hline No. of ED outpatient visits & 24587 & 0.21 & 0.081 \\
\hline No. of general surgeons & 7.03 & -0.20 & 0.101 \\
\hline No. of certified general surgeons & 5.73 & -0.19 & 0.108 \\
\hline No. of blood banks & 0.97 & -0.22 & 0.071 \\
\hline No. of neurosurgeons & 0.04 & -0.09 & 0.479 \\
\hline No. of hospital admissions & 8171 & -0.22 & 0.060 \\
\hline Trauma center $(\mathrm{Y} / \mathrm{N})$ & $8 / 92^{\star}$ & -0.21 & 0.075 \\
\hline ALS (ALS/BLS) & $76 / 24^{\star}$ & -0.34 & 0.003 \\
\hline $911(\mathrm{Y} / \mathrm{N})$ & $59 / 41^{\star}$ & -0.26 & 0.030 \\
\hline
\end{tabular}

pediatric trauma death rates (population density; $R=$ $-0.29, p=0.014$ and total population; $R=-0.21, p$ $=0.020$ ). Rural percentage was found to be correlated positively with per capita pediatric county trauma death rates $(R=0.16, p=0.020)$ (Table 2$)$. The less populated, more rural areas had a higher per capita death rate.

\section{Socioeconomic}

There were four socioeconomic measures of county status tested. The non-Caucasian percent of the population was the only variable associated significantly with pediatric trauma mortality rates $(R=0.24, p=0.040)$.

\section{Environment}

The number of miles of primary highway and the miles of highway exposure were not related to pediatric trauma death rates.

\section{Medical Resources}

Each of the 11 medical resource variables studied correlated negatively with pediatric trauma mortality rates, except the number of emergency department outpatient
Table 3. RESULTS OF MULTIVARIATE ANALYSIS $\left(R^{2}=0.247\right)$

\begin{tabular}{lccc}
\hline \multicolumn{1}{c}{ Variable } & Type III SS & F Value & p Value \\
\hline Percent unemployed & 5.37 & 8.96 & 0.004 \\
ALS & 4.62 & 7.72 & 0.007 \\
Number miles primary highway & 4.56 & 7.62 & 0.007 \\
Rural & 1.59 & 2.65 & 0.107 \\
\hline
\end{tabular}

visits. Only two (presence of ALS and access to 911-telephone service) reached statistical significance $(p=0.003$ and $p=0.030$, respectively). The number of hospital intensive care unit beds was of borderline statistical significance $(p=0.054)$.

\section{Multivariate Analysis of the Association of Per Capita County Pediatric Trauma Death Rates with County Demographic Variables and Medical System Factors}

Univariate analyses identified a number of county descriptors and medical resources that were associated significantly with the per capita pediatric trauma death rates. Ordinary least-squares multiple linear regression was used to evaluate the association of multiple independent variables with the per capita pediatric county trauma death rates. Those variables found to be associated significantly with per capita pediatric county trauma death rates were the unemployment percentage, the presence of ALS, the primary highway mileage, and rurality (Table 3).

\section{Discussion}

Traumatic injury is the fourth leading cause of death in the United States. ${ }^{9-11}$ It is responsible for more deaths than cancer and cardiovascular disease combined. ${ }^{12}$ The awareness of trauma as a public health epidemic has grown in recent years; ${ }^{13}$ this has led to the development of more effective methods of prevention and treatment of injury.

Trauma is also the leading cause of death and disability in children..$^{2-4}$ Injury-related deaths are one of the most important health-care problems affecting the young. However, there is still much that must be done to decrease the number of pediatric traumatic deaths. This study analyzed county pediatric trauma death rates and the factors that can affect them. The development of prevention and treatment programs can be accomplished only through identification and quantification of specific demographic and medical system factors that significantly affect pediatric trauma death rates.

The association of a variety of county demographic 
and medical system factors with the per capita pediatric county trauma death rates in North Carolina were analyzed. Several demographic variables and medical system factors were expected to demonstrate an association with the pediatric mortality rate. We hoped that identifying factors associated with trauma death would aid in the development of trauma systems and the use of resources to provide more effective treatment of pediatric trauma. This information could also be instrumental in developing appropriate injury prevention programs for children.

In this study, children were under-represented in the total population of trauma deaths; children make up $21 \%$ of the total population, but only $7 \%$ of trauma deaths occur in patients younger than age 15 .

In this population-based study, the only medical system resource factor associated significantly with per capita pediatric county trauma death rate was the county level of EMS certification. No other medical resource factors were significant by multivariate analysis, including number of hospital intensive care unit beds, number of hospital discharges, number of emergency outpatient visits, number of general surgeons, number of neurosurgeons, number of hospital admissions, presence of trauma centers, and access to 911-telephone service. However, by univariate analysis, both the presence of ALS and the presence of 911-telephone service were significant.

It has been assumed that ALS does not affect the outcome of trauma. ${ }^{14-16}$ This is the first study that has demonstrated the positive association of ALS with decreasing trauma death rates in children. Such information is important because of the controversy over "scoop and run" versus ALS interventions for pre-hospital care in trauma. ${ }^{17}$ This study suggests that counties with pre-hospital providers who are trained to ALS level experience can decrease per capita pediatric trauma death rates.

Special facilities, equipment, and personnel are necessary to achieve an optimal outcome for the injured child. ${ }^{5}$ Children often receive poor medical care from unprepared and disorganized systems; this can result in death or long-term disability. ${ }^{5}$ Our study findings support the hypothesis that increased levels of training in the pre-hospital care phase can improve outcomes in pediatric trauma. In children with head injuries, the early endotracheal intubation provided by ALS-trained caregivers may be necessary to eliminate airway obstruction and restore breathing. However, our study was not able to demonstrate an association between any other medical system factor and pediatric injury deaths.

Adult emergency medical service systems exist in every region of the United States, but little attention has been paid to the development of emergency medical systems for the injured child. ${ }^{5}$ In response to this problem, pediatric advanced life support (PALS) training for emergency medical technicians has been developed. This training focuses on the special considerations and needs of the pediatric patient in an emergency. Currently, there is insufficient information on PALS and its availability in North Carolina counties. At this stage, it can only be speculated that the presence of PALS training could further reduce per capita pediatric trauma death. When sufficient information becomes available, there should be further study on this subject.

Univariate analysis showed population density, total population, rural percentage, and non-Caucasian population percentage to have a significant association with per capita pediatric county trauma death rates. Multivariate analysis showed rurality, unemployment percentage, and primary highway mileage to have a significant association with per capita pediatric county trauma death rates. The findings of the multivariate analysis suggest that children younger than 15 years are at greater risk for trauma deaths if they reside in a poor, rural county with numerous miles of primary highways and no access to ALS.

The association between the primary highway mileage and per capita county trauma death rates in children may be related to an association between highway miles and deaths due to motor vehicle crashes. Education on preventing motor vehicle crashes and enforcement of laws enacted to prevent accidents (speed limit, seat belt, child restraint, and driving-while-impaired laws) should prevent pediatric trauma deaths.

The associations between rurality and unemployment percentage and per capita pediatric county trauma deaths may be related to a variety of factors. Rural counties with high unemployment often are lower in all measures of socioeconomic status. This low socioeconomic status may impact on the type of medical resources available in the county, the quality of the resources available, and the type of treatment that the patient seeks. Other studies have indicated similarly that pediatric and adult trauma deaths are more likely to occur in rural areas rather than in urban areas. ${ }^{19,20}$

Males were over-represented in the sample of trauma deaths in this study. Although in North Carolina the male population younger than 15 years is approximately $51 \%, 64 \%$ of trauma deaths in children younger than 15 years between 1986 and 1989 were in boys. Although patterns of fatal trauma differ among countries, the high incidence of trauma among boys has been observed in many countries. ${ }^{21}$ Further study into the causes of increased trauma in male children is necessary.

Although African Americans represent 23\% of the North Carolina population, they accounted for $40 \%$ of all deaths due to injury in children younger than 15 years. Deaths and disability due to injury occur disproportionately among poor people, many of whom belong to minority groups. ${ }^{1}$ Studies that identify the race- and 
cause-specific risk factors that predispose African American children to higher death rates due to injury are necessary to reduce this incidence. ${ }^{1}$ Injury prevention programming should be targeted at both the African American and male pediatric populations.

An unpublished study by our group on trauma death rates in total populations indicated that various factors are associated with per capita trauma death rates. In this study, univariate analysis revealed that some factors associated with per capita trauma death rates in the total population are also associated with per capita trauma death rates in children. However, some variables differed in significance between the two populations. For example, the presence of a trauma center in the county had no significant association with per capita pediatric trauma death rates in North Carolina counties, but it was associated significantly with the per capita trauma death rates in the population as a whole. ${ }^{20}$ In a study of trauma death rates in the elderly, the only variable found to be associated significantly with elderly mortality rates of North Carolina is the non-Caucasian population percentage. ${ }^{22}$

The results of multivariate analysis of pediatric trauma and trauma in populations as a whole differed. Percent unemployment and the presence of ALS were the only two variables that were associated highly with per capita trauma deaths in both populations. The presence of a trauma center in the county again was associated significantly with per capita trauma death rates in the population as a whole, but not with per capita trauma death rates in children.

Although there is a common association of county descriptors, such as measures of rurality and socioeconomic status with per capita trauma death rates in both adult and pediatric populations, the types of medical system resources that are associated with decreased per capita trauma death rates vary between the two groups. Pediatric trauma should be distinguished from adult trauma in its relationship to medical system factors. Many county descriptors are associated with an increase or decrease in the risk of death from pediatric trauma. This information can aid government agencies and health-care providers in using their resources in a more efficient and economic manner. The expansion of the availability of ALS, particularly in rural counties, may lower significantly the per capita pediatric county trauma death rate. Pediatric trauma is a serious healthcare problem, and this study suggests a need for improvement of prevention and treatment programs for pediatric trauma through appropriate medical system resource adjustments.

\section{REFERENCES}

1. Gulaid JA, Onwuachi-Saunders EC, Sacks JJ, et al. Differences in death rates due to injury among blacks and whites. JAMA 1989 261:214-216.

2. Haller, JA. Pediatric trauma, no. 1 killer of children. JAMA 1983; 249:47.

3. Luten RC. The child in the EMS system. Pediatrics 1990; 85:879887.

4. Vane D, Shedd FG, Grosfeld JL, et al. An analysis of pediatric trauma deaths in Indiana. J Pediatr Surg 1990; 25:955-959.

5. Ramenofsky ML. Emergency medical services for children and pediatric trauma system components. J Pediatr Surg 1989; 24:153155.

6. Baker S, Whitfield P, O'Neill B. County mapping of injury mortality. J Trauma 1988; 28:741-745.

7. Baker $S$, Whitfield $P, O$ 'Neill B. Geographic variations in mortality from motor vehicle crashes. N Engl J Med 1987; 316:1384-1387.

8. Cox J. Risks to health in rural areas. Practitioner 1983; 227:14731745.

9. Baker S, O’Neill B, Karpf RS. The Injury Fact Book. Lexington, MA.: Lexington Books, 1984; p7.

10. Committee on Trauma Research. Injury in America. Washington, DC: National Academy Press, 1985; p1.

11. U.S. Preventative Services Task Force. Counseling to prevent motor vehicle injuries. Am Fam Phys 1990; 41:1465-1470.

12. Cotten P. Preventive medicine extends to injuries, too. JAMA 1990; 263:2597.

13. Oller DW, Maxwell JG, Clancy TV. Trauma care in NC: an update. NC Med J 1990; 51:463-464.

14. Gervin AS, Fischer RP. The importance of prompt transport in salvage of patients with penetrating heart wounds. J Trauma 1982; 22:443.

15. Mattox KL, Bickell W, Pepe PE, et al. Prospective Mast study in 911 patients. J Trauma 1989; 29:1104.

16. Smith JP, Bodai BI, Hill AS, et al: Pre-hospital stabilization of critically injured patients: a failed concept. J Trauma 1989; 25:65.

17. Messick WJ, Rutledge R, Meyer AA. The association of advanced life support training and decreased per capita trauma death rates: an analysis of 12,417 trauma deaths. J Trauma (in press). Presented at the Eastern Association of Surgeons in Trauma meeting, January 1991.

18. Ornato JP, Racht EM, Fitch JJ, et al. The need for ALS in urban and suburban EMS systems. Editorials. Ann Emerg Med 1990; 19: 1469-1470.

19. Nakayama DK, Gardener MJ, Rowe MI. Emergency endotracheal intubation in pediatric trauma. Ann Surg 1990; 211:218-223.

20. Rutledge R, Messick J, Baker CC, et al. Multivariate population based analysis of the association of county trauma centers with per capita county trauma death rates. J Trauma 1992; 33:29-38.

21. Honkanen R, Koivumaa-Honkanen H, Smith G. Males as a highrisk group for trauma: the Finnish experience. J Trauma 1990; 30: 155-162.

22. Martin G, Rutledge R. A Study of Trauma Death in the Elderly. Unpublished study, University of North Carolina, 1991. 